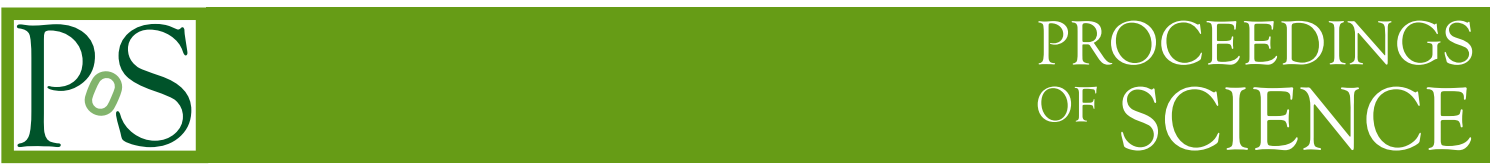

\title{
Field and matter or pure field physics?
}

\author{
I.E. Bulyzhenkov* \\ Moscow Institute of Physics and Technology, Moscow, 115184, Russia \\ E-mail: ibphys@gmail.com
}

\begin{abstract}
We completely share the 1938 Einstein's approach "What impresses our senses as matter is really a great concentration of energy into a comparatively small space..." and "We could regard matter as being made up of regions of space in which the field is extremely intense. . . There would be no place in this new physics for both field and matter, for the field would be the only reality." In our developments, the scalar Ricci curvature in pseudo-Riemannian space-time represents local densities elementary continuous masses within everywhere nonempty space. Infinitely extended particles with their $r^{-4}$ radial mass densities form observable bodies and obey Newtonian attractions in weak fields but repulsions in strong ones. Material space of $r^{-4}$ overlapping radial monopoles is also filled by unobservable $r^{-2} \times r^{-2}$ bi-pole distributions of dark mass-energy. The weak field radial fall in such a space with dark energy would ultimately lead to strong field regions with gravitational repulsion and deceleration of probe bodies. This strong field repulsion quantitatively relates the Hubble expansion speed $r H_{o}$ to the Metagalaxy mass and predicts $r\left(H_{o}\right)^{2}$ acceleration rate of the expanding Universe. Global oscillations of the world material space around its equilibrium state at equal bi-pole and monopole energies are predicted in a line of Penrose cyclic cosmology.
\end{abstract}

Frontiers of Fundamental Physics 14 - FFP14,

15-18 July 2014

Aix Marseille University (AMU) Saint-Charles Campus, Marseille

\footnotetext{
* Speaker.
} 


\section{Introduction into Einstein's pure field physics and metric $g_{o o}$-theorem}

In 1938 Einstein and Infeld very clearly formulated science directives for further evolution of physics [1]:

- "Matter is where the concentration of energy is great, field where the concentration of energy is small. But if this is the case, then the difference between matter and field is a quantitative rather than a qualitative one;"

- "We could regard matter as being made up of regions of space in which the field is extremely intense. . . There would be no place in this new physics for both field and matter, for the field would be the only reality;"

- "A thrown stone is, from this point of view, a changing field, where the states of greatest field intensity travel through space with the velocity of the stone. There would be no place, in our new physics, for both field and matter, field being the only reality;"

- "Our ultimate problem would be to modify our field laws in such a way that they would not break down for regions in which the energy is enormously concentrated;"

- "A coherent field theory requires that all elements be continuous ... And from this requirement arises the fact that the material particle has no place as a basic concept in a field theory. Thus, even apart from the fact that it does not include gravitation, Maxwell's theory cannot be considered as a complete theory."

There were no mathematical errors in 1916 Schwarzschild's solution for curved metric of empty space [2], but Einstein was disappointed by the discontinuous matter concept in general and ultimately inferred pure field physics without (point) particles at all. Called lately 'the reluctant father of black holes', he firmly stated from the 1939 thought experiment [3] that "Schwarzschild singularities are not relevant to physical reality".

Einstein's metric theory of continuous material space without particles is to be newly analyzed in a self-contained form in order to avoid the conceptual shortage of the Newton attraction of spatially separated point particles in empty space. Despite Newton employed for gravitation localized masses in empty space, material sources in the Einstein Equation are stress-energy tensor densities but not scalar mass invariants. Energy density type of gravitational sources is more suitable for a continuous distribution of the extended elementary mass-energy rather than for a point mass singularity. In other words, Newtonian empty-space references cannot be accepted in principle (or by default) by Einstein's metric gravitation of overlapping mass-energy charges in joint material space.

In a line of Einstein's pure field approach to observable bodies, it is essential to employ metric references for General Relativity (GR) not on the basis of the Newton empty space theory, but on a self-contained basis like the Special Relativity (SR) limit for GR energy of a probe body. In favor of such coherent self-references, Einstein's metric formalism [4] uniquely relates the forth component,

$$
P_{o} \equiv m c g_{o \mu} \frac{d x^{\mu}}{d s} \equiv m c\left(g_{o o} V^{o}+g_{o i} V^{i}\right) \equiv \frac{m c \sqrt{g_{o o}}}{\sqrt{1-v^{2} c^{-2}}} \equiv \frac{(K+U)}{c},
$$

of the covariant four-momentum $P_{\mu} \equiv m c g_{\mu \nu} d x^{v} / d s$ of the probe scalar mass $m$ to its full relativistic energy $E=K+U$ containing positive kinetic energy $K=m c^{2} / \sqrt{1-v^{2} c^{-2}}$ and negative 
potential energy $U$ associated with gravitational interactions. One can use the GR energy definition $E \equiv c P_{o}>0$ in order to rewrite $g_{o o}$ metric component in terms of the negative gravitational potential $U / c P_{o}$ for GR energy-charge $E$, which is the only measure of inertia (and gravity) of the probe mass $\mathrm{m}$ :

$$
\sqrt{g_{o o}} \equiv(K+U) \frac{\sqrt{1-v^{2} c^{-2}}}{m c^{2}} \equiv 1+\frac{U \sqrt{g_{o o}}}{E} \equiv \frac{1}{[1-(U / E)]} .
$$

We used (1.1) relations to perform identical algebra transformations in (1.2). The latter equalities proofs the following metric $g_{o o}$-theorem: "Time-time component of the pseudo-Riemann metric tensor in Einstein's GR is defined by a gravitational field potential $\varphi=U / E$ exactly as $g_{o o}=(1-\varphi)^{-2}$, which has no peculiarities for $-\infty<\varphi \leq 0$ ". It is following from (1.2) that Schwarzschild [2] empty-space metrics (where $g_{o o}=\left[1-\left(2 G M / c^{2} r\right)\right]$ for weak and strong fields) do not match the metric $g_{o o}$-theorem and, consequently, the Einstein energy definition in strong gravitational fields. Indeed, Newtonian point mass gravitation is not a true limit for interaction of overlapping energy charges in Einstein's pure field relativity.

\section{Energy balance of gravitating monopoles and dark bi-poles in joint material continuum}

Contrary to Schwarzschild's metric with singularities, criticized by Einstein [3], GR metric solutions $[5,6,7]$ of static non-empty space with $g_{o o}=(1-\varphi)^{-2}$ exhibit only smooth analytical functions due to inherent symmetries $\gamma_{i j}(\varphi) \equiv g_{o i} g_{o j} g_{o o}^{-1}-g_{i j}=\delta_{i j}$ of pseudo-Riemannain manifolds applied to real matter. Pure field physics of extended continuous particles describes [8] main relativistic tests and observations much more self-consistently than the traditional point source physics which is unable to interpret the scalar Ricci curvature as the geometrized mass density.

Curved empty space has not provided yet an exact metric solution for the many-body gravitating system, while analytical metric solution for flat non-empty space of overlapping mass-energies has been already found [6]. Mechanical (inertial or passive, $\mu_{p} c^{2}$ ) and gravitational (potential or active, $\mu_{a} c^{2}$ ) energy densities of static non-empty space depend only on the relativistic logarithmic potential (or the local tension of material space),

$$
W(\mathbf{x}) \equiv-c^{2} \ln \frac{1}{\sqrt{g_{\text {oo }}(\mathbf{x})}}=-c^{2} \ln \left(1+\frac{r_{1}}{\left|\mathbf{x}-\mathbf{a}_{1}\right|}+\frac{r_{2}}{\left|\mathbf{x}-\mathbf{a}_{2}\right|}+\ldots+\frac{r_{n}}{\left|\mathbf{x}-\mathbf{a}_{n}\right|}\right) .
$$

Here $r_{i} \equiv G E_{i} / c^{4}=G m_{i} / c^{2}$ is Schwarzschild-type coordinate scale of the elementary energycharge $E_{i}$ (distributed everywhere in 3-space but mainly in the vicinity of dense-energy centers at $\mathbf{a}_{i}$ ). The inhomogeneous tension (2.1) defines GR time delay $\sqrt{g_{o o}} d x^{o}$ of a local observer and complies with the Einstein Principle of Equivalence for passive and active mass densities,

$$
\mu_{p}(\mathbf{x}) \equiv \frac{[-\nabla W(\mathbf{x})]^{2}}{4 \pi G c^{2}}=\frac{\nabla^{2} W(\mathbf{x})}{4 \pi G} \equiv \mu_{a}(\mathbf{x}) .
$$

Now, by using (2.2), we prove strict conservations of whole (gesamt) active and passive mass-energies of any metric non-empty space with the continuous Ricci curvature $g^{\mu v} R_{\mu v}=$ 


$$
\begin{aligned}
& \left(\mu_{p}+\mu_{a}\right) 8 \pi G / c^{2}>0 \\
& E_{\text {gesamt }}=\int \mu_{p} c^{2} d^{3} x \equiv \frac{c^{4}}{4 \pi G} \int\left(\frac{\frac{\left(\mathbf{x}-\mathbf{a}_{1}\right) r_{1}}{\left|\mathbf{x}-\mathbf{a}_{1}\right|^{3}}+\frac{\left(\mathbf{x}-\mathbf{a}_{2}\right) r_{2}}{\left|\mathbf{x}-\mathbf{a}_{2}\right|^{3}}+\ldots+\frac{\left(\mathbf{x}-\mathbf{a}_{n}\right) r_{n}}{\left|\mathbf{x}-\mathbf{a}_{n}\right|^{3}}}{1+\frac{r_{1}}{\left|\mathbf{x}-\mathbf{a}_{1}\right|}+\frac{r_{2}}{\left|\mathbf{x}-\mathbf{a}_{2}\right|}+\ldots+\frac{r_{n}}{\left|\mathbf{x}-\mathbf{a}_{n}\right|}}\right)^{2} d^{3} x \equiv E_{\text {mono }}+E_{b i} \\
& \quad=\int \frac{c^{4} R d^{3} x}{16 \pi G}=\int \mu_{a} c^{2} d^{3} x \equiv \frac{c^{2}}{4 \pi G} \oint_{\infty}(-\nabla W) d \mathbf{S}=\left(m_{1}+m_{2}+\ldots+m_{n}\right) c^{2}=\text { const }
\end{aligned}
$$

Notice that the integral mass-energy (2.3) of joint non-empty space with the inhomogeneous metric tension (2.1) of overlapping radial masses $m_{i}$ is independent from particular positions $\mathbf{a}_{i}$ of their heavy centers. Such a universal conservation for a system of overlapping radial monopoles under negative gravitational potentials can take place due to hidden (from direct observations) energy contributions into paired (bi-pole) formations of material densities in (2.3).

The upper line in (2.3) sheds new light on the origin of GR potentials $\sqrt{g_{o o}^{\neq i}\left(\mathbf{a}_{i}\right)}$ of radial masses $m_{i}$ (observed in practice at $\mathbf{a}_{i}$ ). For the most of applications, one may always use $\left|\mathbf{a}_{k}-\mathbf{a}_{i}\right| \equiv$ $R_{i k}>>r_{i}+r_{k}=G\left(m_{i}+m_{k}\right) / c^{2}$ for distances between centers of different radial monopoles,

$$
\begin{gathered}
E_{\text {mono }} \approx \int \frac{d^{3} x c^{4} r_{1}^{2} / 4 \pi G}{|\mathbf{x}|^{4}\left(1+\frac{r_{1}}{|\mathbf{x}|}+\frac{r_{2}}{\left|\mathbf{a}_{1}-\mathbf{a}_{2}\right|}+\ldots+\frac{r_{n}}{\left|\mathbf{a}_{1}-\mathbf{a}_{n}\right|}\right)^{2}}+\int \frac{d^{3} x c^{4} r_{2}^{2} / 4 \pi G}{|\mathbf{x}|^{4}\left(1+\frac{r_{1}}{\left|\mathbf{a}_{2}-\mathbf{a}_{1}\right|}+\frac{r_{2}}{|\mathbf{x}|}+\ldots+\frac{r_{n}}{\left|\mathbf{a}_{2}-\mathbf{a}_{n}\right|}\right)^{2}} \\
\ldots+\int \frac{d^{3} x c^{4} r_{n}^{2} / 4 \pi G}{|\mathbf{x}|^{4}\left(1+\frac{r_{1}}{\left|\mathbf{a}_{n}-\mathbf{a}_{1}\right|}+\frac{r_{2}}{\left|\mathbf{a}_{n}-\mathbf{a}_{2}\right|}+\ldots+\frac{r_{n}}{|\mathbf{x}|}\right)^{2}}=c^{2} \sum_{i=1}^{n} m_{i} \sqrt{g_{o o}^{\neq i}\left(\mathbf{a}_{i}\right)} \approx \sum_{i=1}^{n} m_{i}\left(c^{2}-\sum_{k \neq i}^{n} \frac{G m_{k}}{R_{i k}}\right)>0 .
\end{gathered}
$$

Here all monopole energies, $E_{2} \equiv c^{2} m_{2} \sqrt{g_{o o}^{\neq 2}\left(\mathbf{a}_{2}\right)} \equiv c^{2} m_{2} /\left(1+\frac{r_{1}}{\left|\mathbf{a}_{2}-\mathbf{a}_{1}\right|}+\frac{r_{3}}{\left|\mathbf{a}_{2}-\mathbf{a}_{3}\right|}+\ldots+\frac{r_{n}}{\left|\mathbf{a}_{2}-\mathbf{a}_{n}\right|}\right)$ for example, contain negative potential shifts which lead in weak fields to Newtonian attractions of observable radial matter. Negative potentials of gravitating monopoles in (2.4) do not mean decrease of the whole system mass-energy (2.3), because paired attractions are always accompanied by (dark) energy deposits into unobservable bi-pole distributions,

$$
E_{b i}=\frac{c^{4}}{4 \pi G} \sum_{i=1}^{n} \sum_{k \neq i}^{n} \int_{0}^{2 \pi} d \varphi \int_{0}^{\infty} r^{2} d r \int_{0}^{\pi} \frac{\pi_{i} r_{k}\left(r^{2}-R_{i k} r \cos \theta\right) \sin \theta d \theta}{r^{3}\left(R_{i k}^{2}+r^{2}-2 R_{i k} r \cos \theta\right)^{3 / 2}} \approx \sum_{i=1}^{n} \sum_{k \neq i}^{n} \frac{G m_{i} m_{k}}{R_{i k}}>0 .
$$

Notice that gravitating radial monopoles carry only positive energies in (2.3)-(2.4). And bi-polar, dark fields also take only positive energies. The latter have to balance the constant Ricci massenergy (2.3) or the integral GR action of the whole material continuum. In this coherent approach there are no net negative energies at all behind gravitational interactions. Gravitation is not an infinite decrease of negative potentials, as in the Newton theory, but is the universal tendency of a free mechanical system toward equilibrium with the equipartition distribution of energy between available degrees of freedom associated with (observable) monopoles and (dark) bi-poles. Recall that dark matter and dark mass-energy have the same meaning in pure field physics.

\section{Accelerated Hubble expansion and gravitational repulsion in strong fields}

Dynamics of probe bodies in material continuum of overlapping radial masses [5, 6, 7] not only explains all known GR tests [8], but also predicts that the nonempy space paradigm can 
be distinguished from the empty space approach by observing slightly different clock rates in gravitational fields. Indeed, precise measurements of the gravitational time dilation in the EarthSun-Moon system with the time varying Newton potential $\varphi$ can be compared with predictions $(d \tau-d t) / d t \equiv \sqrt{g_{o o}}-1 \approx \varphi\left(1-\varphi / 2 c^{2}\right) / c^{2}$ of the Schwarzschild metric [2] for point mass physics and with the temporal redshift $\sqrt{g_{o o}}-1 \approx \varphi\left(1+\varphi / 2 c^{2}\right) / c^{2}$ in non-empty space due to (1.2).

Again, material space continuum in Einstein's GR metric formalism always keeps Euclidean 3D subgeometry due to inherent symmetries [5] of curved 4D geometry. GR geodesic equations of motions in pseudo-Riemann space-time with $0 \leq g_{o o} \leq 1$ and flat 3D intervals, $g_{o i} g_{o j} g_{o o}^{-1}-g_{i j}=\delta_{i j}$, have been derived [8] for strong static fields in the following form,

$$
\left\{\begin{array}{l}
g_{o o} d t / d p=1, d p / d s=g_{o o} d t / d s=E_{m} / m=\text { const } \\
r^{2} d \varphi / d p=J_{\varphi}=\text { const } \\
r^{2} d \varphi / d s=J_{\varphi} E_{m} / m \equiv L=\text { const } \\
(d r / d p)^{2}+\left(J_{\varphi} / r\right)^{2}-g_{o o}^{-1}=\text { const }\left(=-m^{2} / E_{m}^{2}\right) \\
(d r / d s)^{2}+(r d \varphi / d s)^{2}-E_{m}^{2} / m^{2} g_{o o}=-1,
\end{array}\right.
$$

where GR energy $E_{m}$ and angular momentum $J_{\varphi}$ are first integrals of the probe body relativistic motion and $m=$ const is the probe body scalar mass. GR speed of the radial fall from infinity,

$$
d r / d t= \pm c \sqrt{g_{o o}\left(1-g_{o o}\right)},
$$

can be expressed through the world time $d x^{o}=d t$ of a distant observer, because one always can use in (3.1) $E_{m} / m \equiv c^{2} \sqrt{g_{o o}} / \sqrt{1-v^{2} c^{-2}}=$ const $\Rightarrow 1, d \varphi / d s=0, d s=\sqrt{g_{o o}} c d t \sqrt{1-v^{2} c^{-2}}$, and $v^{2}=\left(d r / \sqrt{g_{o o}} d t\right)^{2}$. Metric (1.2) for one gravitating center, $g_{o o}=1 /\left[1+\left(r_{o} / r\right)\right]^{2}$ with $r_{o}=G M / c^{2}$, leads in (3.2) to (unstable) motionless states, $d r / d t=0$, of small probe masses at final stages of their radial falls. The probe mass reaches maximum radial speed $d r / d t=c / 2$ of the central fall at $r=r_{o}(1+\sqrt{2})$. The decelerating path of the radial fall takes place below $r_{o}(1+\sqrt{2})$ due to gravitational repulsion of strong fields.

Coordinate acceleration $d^{2} r / d t^{2}$ can be derived from (3.2) by taking its time derivative,

$$
d^{2} \mathbf{r} / d t^{2}=-c^{2} r_{o} \mathbf{r}\left(r^{2}-2 r_{o} r-r_{o}^{2}\right) /\left(r+r_{o}\right)^{5},
$$

which universally describes the Newton attraction $-r_{o} c^{2} \mathbf{r} / r^{3}$ for $r_{o} \ll r$ and the strong-field GR repulsion $+\mathbf{r} c^{2} / r_{o}^{2}$ for $r \ll r_{o}$. Attraction acceleration in (3.3) takes its extreme value $9.2 \times 10^{-3} c^{2} / r_{o}$ at $4.48 r_{o}$, while repulsion acceleration takes its maximum $0.12 c^{2} / r_{o}$ at $0.35 r_{o}$. Recall that both repulsion and attraction of probe masses corresponds to their motion in negative gravitational potentials (2.1) and positive Ricci mass densities (2.2).

Nowadays the gravitational scale $R_{o}=G M_{\text {Meta }} / c^{2}$ of our Metagalaxy is still higher than its observable dimension. According to (3.3), such a dense Metagalaxy should repeal its material elements behind $R_{o}(1+\sqrt{2})$. The strong-field limit of (3.2), when $r \ll R_{o}$ and $d r / d t=c r / R_{o} \Rightarrow r H_{o}$, corresponds to the Hubble law of expanding galaxies at $R_{o} \Rightarrow c / H_{o}=1,3 \times 10^{26} \mathrm{~m}$. The Hubble constant $2,3 \times 10^{-18} \mathrm{sec}^{-1}$ predicts from (3.2) $M_{\text {Meta }}=R_{o} c^{2} / G=1.8 \times 10^{53} \mathrm{~kg}$. These computations fully correspond to evaluations of $M_{\text {Meta }}$ from other observations. The Universe expansion acceleration, $d^{2} r / d t^{2}=c^{2} r / R_{o}^{2} \Rightarrow r H_{o}^{2}$, in the considered limit $r \ll R_{o}$ is also proportional to the distance $\mathrm{r}$. Therefore, the Hubble expansion with acceleration reads for material continuum of our dense Metagalaxy as 


$$
V(r)=\left(1+t H_{o}\right) r H_{o}=\left[1+\frac{t c^{3}}{G M_{\text {Meta }}}\right] \frac{r c^{3}}{G M_{\text {Meta }}},
$$

where $H_{o}^{-1}$ defines the world expansion time and, therefore, $t H_{o} \leq t_{\max } H_{o}=1$.

\section{Conclusions}

Centers of (extended) massive bodies in the Earth laboratory are always separated by huge distances compared to bodies'gravitational scales and, therefore, dark interference or bi-pole energy deposits of laboratory bodies are very-very small compared to their mass-energies. Massive galaxies have detectable gravitational scales and contemporary observations of matter near a galaxy center can provide relevant data to elicit bi-pole (dark) mass-energy deposits. The extremely dense region in the center of our galaxy is larger than its gravitational scale. And diameters of neutron stars are always above their gravitational scales as is known. Strong field gravitational repulsion rather than the black hole horizon should be applied to physics of these dense energy regions.

A mega system with gravitational attraction/repulsion of monopoles in negative potential keeps due to (2.3) Ricci mass-energy conservation for all visible matter together with dark (bi-pole) deposits. The Big Bang fragmentation of one radial monopole into a system of expanding (with acceleration) radial monopoles and dark bi-poles corresponds to (3.2)-(3.3) and to the aforementioned tendency to equipartition distribution of energy between monopole and bi-pole degrees of freedom. Now visible matter of the Metagalaxy in whole is still strongly concentrated with high excess of dark energy that results in the world expansion with acceleration. One day the Universe pendulum of 80 billions of galaxies with constant Ricci mass-energy (2.3) will enter into the predicted [9] contraction cycle for the spatial material continuum. The equilibrium configuration of oscillating non-empty space is to be next to equipartition distribution of dark (bi-pole) and observable (monopole) energy contents.

\section{References}

[1] A. Einstein and L. Infeld, The Evolution of Physics (Simon and Schuster, N.Y., 1954), 256.

[2] K. Schwarzschild, Sitzungsber. Deut. Akad. Wiss., Berlin (1916) 189.

[3] A.Einstein, Annals of Mathematics 40 (1939) 922.

[4] A. Einstein, Annalen der Physik 49 (1916) 769.

[5] I.E. Bulyzhenkov, Int. J. Theor. Physics 47 (2008) 1261.

[6] I.E. Bulyzhenkov, Jour. Supercond. and Novel Magn. 22 (2009) 723.

[7] I.E. Bulyzhenkov, Bullet. Lebedev Phys. Inst. 21 (2014) 1.

[8] I.E. Bulyzhenkov, Jour. Mod. Physics 3 (2012) N.10, 1465.

[9] R. Penrose, Cycles of Time: An Extraordinary New View of the Universe (Bodley Head, 2010, ISBN 978-0-224-08036-1) 